\title{
Erratum to: Loss of Pericytes in Radiation Necrosis after Glioblastoma Treatments
}

\author{
Soon-Tae Lee ${ }^{1} \cdot$ Youngbeom Seo $^{2} \cdot \mathrm{Ji}^{-Y e o n} \mathrm{Bae}^{1} \cdot \mathrm{Kon} \mathrm{Chu}^{1} \cdot \mathrm{Jin}$ Wook Kim ${ }^{3}$. \\ Seung Hong Choi ${ }^{4}$. Tae Min Kim ${ }^{5}$ - Il Han Kim ${ }^{6}$ • Sung-Hye Park ${ }^{7}$. Chul-Kee Park ${ }^{3}$ (D)
}

Published online: 9 September 2017

(C) Springer Science+Business Media, LLC 2017

\section{Erratum to: Molecular Neurobiology}

https://doi.org/10.1007/s12035-017-0695-Z

The original version of this article unfortunately contained an error on the Acknowledgements. The author missed to correct the Grant number from SNUH Research Fund (0420150850) when it should be "SNUH Research Fund (0420160640)".

The online version of the original article can be found at https://oi.org/ $10.1007 / \mathrm{s} 12035-017-0695-\mathrm{Z}$

Chul-Kee Park

nsckpark@snu.ac.kr

1 Department of Neurology, Seoul National University Hospital, Seoul, Korea

2 Department of Neurosurgery, Yeungnam University College of Medicine, Daegu, Korea

3 Department of Neurosurgery, Seoul National University Hosptial, Seoul National University College of Medicine, Seoul, Korea

4 Department of Radiology, Seoul National University Hospital, Seoul National University College of Medicine, Seoul, Korea

5 Department of Internal Medicine, Seoul National University Hospital, Seoul, Korea

6 Department of Radiation Oncology, Seoul National University Hospital, Seoul National University College of Medicine, Seoul, Korea

7 Department of Pathology, Seoul National University Hospital, Seoul National University College of Medicine, Seoul, Korea
The authors hereby publish the corrected Acknowledgements as stated below.

Acknowledgements This research was supported by the National Research Foundation of Korea (NRF) grants funded by the Ministry of Science, ICT \& Future Planning, Republic of Korea (2016M3C7A1914002 by the Brain Research Program, and 2016R1C1B2011815). S.-T.L. was supported by the SNUH Research Fund (0420160640). We are thankful for the artwork done by Mijin Jung. 